\title{
Persistent SARS-CoV-2 infections in Immunocompromised hosts contribute to new variants
}

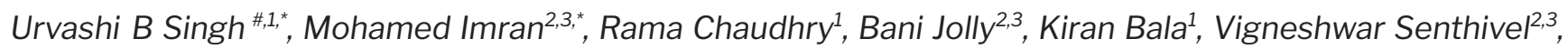
Abhinav Srivastav ${ }^{1}$, Mercy Rophina ${ }^{2,3}$, Raunak Bir ${ }^{1}$, Rahul C. Bhoyar ${ }^{2}$, Lata Kumari ${ }^{1}$, Arvinden VR ${ }^{2,3}$, Nayer Jamshed ${ }^{4}$, Mohit Kumar Divakar, ${ }^{2,3}$, Praveen Aggarwal ${ }^{4}$, Afra Shamnath ${ }^{2}$, Subrata Sinha ${ }^{5}$, Vishu Gupta ${ }^{2,3}$, Anjali Bajaj ${ }^{2,3}$, COVID CBNAAT CORE GROUP ${ }^{6}$, Sridhar Sivasubbu ${ }^{2,3}$, Vinod Scaria ${ }^{2,3}$, Randeep Guleria ${ }^{7}$

${ }^{1}$ Department of Microbiology, All India Institute of Medical Sciences, New Delhi

${ }^{2}$ CSIR-Institute of Genomics \& Integrative Biology, Mathura Road, New Delhi 110025, India

3 Academy for Scientific and Innovative Research, Human Resource Development Centre Campus, Ghaziabad 201 002, Uttar Pradesh, India

${ }^{4}$ Department of Emergency Medicine, All India Institute of Medical Sciences, New Delhi

${ }^{5}$ Department of Biochemistry, All India Institute of Medical Sciences, New Delhi

${ }^{6}$ CBNAAT CORE GROUP

${ }^{7}$ Department of Pulmonary, Critical Care \& Sleep Medicine, All India Institute of Medical Sciences, New Delhi

* Contributed Equally

\#Address for correspondence:

Dr. Urvashi B. Singh MD PhD FAMS

Professor

Department of Microbiology

All India Institute of Medical Sciences,New Delhi-110029

Mobile No. +919811120203

Fax: +911126588663

Email : drurvashi@gmail.com 
Reinfections due to SARS-CoV-2 have been reported from several countries. Repeat positive RT-PCR reports of COVID-19 could be attributed to reinfections or reactivations. Several studies have reported repeat positives ${ }^{1}$. Our study discusses a case series of patients with chronic immunosuppressive illness, who had repeat positive RT-PCR for COVID-19, detected as reinfection and reactivation on whole-genome sequencing.

Patients presenting/following-up for ongoing treatment in a tertiary care centre were tested for SARS-CoV-2 when symptomatic or before an intervention by Cartridge/Chip based Real-time-PCR kits, Genexpert (Cepheid, Sunnyvale, CA) and TrueNat (Molbio Diagnostics, India). Samples from patients found positive for COVID-19 when tested after 28 days were further subjected to whole-genome sequencing. RNA isolated from nasopharyngeal swabs were sequenced as per COVIDSeq protocol ${ }^{2}$ on NovaSeq 6000 sequencing platform (Illumina Inc.) to generate $100 \times 2$ paired end reads. Customized protocols were used to analyse the sequencing data by systemically aligning the raw read data to the reference genome ${ }^{2}$. Variants were called using VarScan (v2.4.4) ${ }^{3}$ at a minimum depth of 5 reads and a minimum frequency of $50 \%$. Lineages were assigned to the genome sequences based on the presence of genetic variations using pangolin (2.4.2, pangoLEARN version 2021-05-12) ${ }^{4}$.

Of over 10,000 patients tested for COVID-19 from the Emergency Department/ before Emergency intervention till August 2020, six patients with repeat positive report for COVID-19 after 28 days of initial diagnosis by Real-time PCR, were identified in retrospect with long standing illness. Six included two patients with Chronic Liver Disease (following Alcoholic Cirrhosis), one patient each with Chronic Kidney Disease, HIV infection, Hypothyroidism and Diabetes mellitus. Clinical timelines of the patients are summarised in Figure 1a.

Genomic analysis of the samples revealed the presence and absence of unique genetic variations between episodes of infection. Unique variants were observed during the second episode of infection in a total of 5 samples. Samples A2, C2, D2, G2 and H2 possessed 18, 2, 3, 7 and 4 unique mutations respectively in comparison to initial infection episodes. Of the six patients, one pair of sample B1 \& B2 was found to be a potential case of reactivation/persistence. All observed genetic variants per episode for each sample is shown in Figure 1b. D138Y, a potential immune evasion variant, located in the $\mathrm{N}$ terminal domain of the Spike protein, was identified in both infection episodes of sample B (B1 \& B2) and in episode 1 of samples C and G (C1 and G1). 
(a)

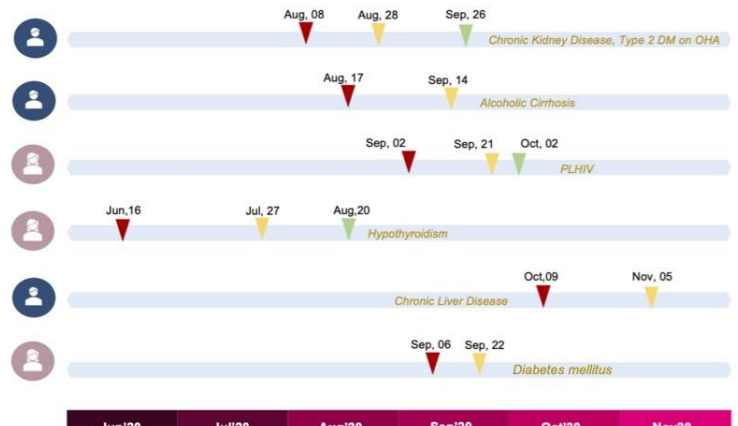

8 Patient-Male $\boldsymbol{\nabla}$ Infection-Episode $1 \quad \nabla$ Infection-Episode 3 (b)
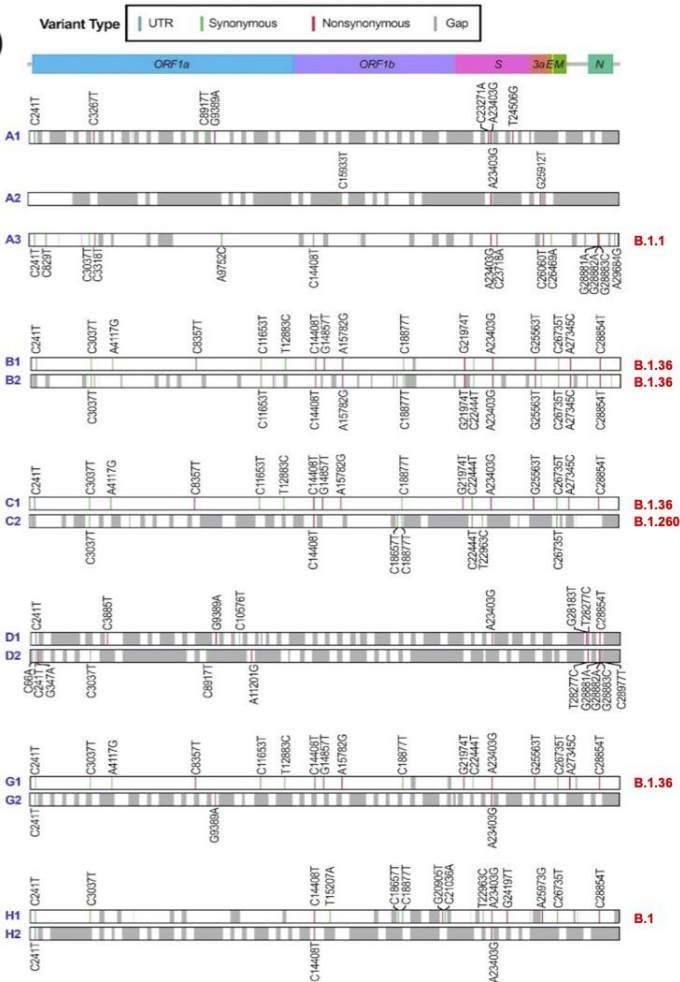

Figure 1. Schematic representation of the clinical timelines of the patients and genomic architecture of the viral genomes isolated and analysed in the study. (a) Clinical timelines of the patients, time of onset of infection episodes and clinical diagnosis. (b) Summary of genome variants present in SARS-CoV-2 isolates of the cases along with their assigned lineages. A potential immune evasion variant, D138Y was identified in both infection episodes of sample B (B1 \& B2) and in episode 1 of samples $C$ and $G$ (C1 and G1). No lineages were assigned for sample $D, A 1, A 2, G 2$ and $H 2$ using PANGOLIN (pangoLEARN version 2021-05-12)

Persistence and severity of SARS-CoV-2 have been found associated with various immunosuppressed conditions and immune dysregulations ${ }^{5}$. Susceptibility to infection and course of infection might also be negatively affected by thyroid dysfunction which adversely affects both innate and adaptive immune system, specifically $T$ cell mediated immune response leading to more severe COVID-19 disease course and prolonged shedding of virus ${ }^{6}$.

Our genomic analysis on samples with repeat positive results revealed that such events, though rare, are not uncommon. These recurrent infections can either be a reactivation/persistence by the same virus or reinfection by a genetically distinct virus. Reinfections have been reported in immune-competent individuals but immunocompromised populations are at a higher risk of serious complications due to poor humoral and cellular immune response ${ }^{7}$. One of the cases which was identified as a potential reactivation/persistence in our study, with Alcoholic liver cirrhosis, reiterates the possibility of persistence and prolonged shedding of COVID-19 in 
immunocompromised patients ${ }^{8}$. Studies have shown that multi-mutational variants could arise during such persistent infections. ${ }^{9}$

The finding of immune evasion mutation in three patients during the initial disease episode suggests that immunocompromised patients could be hosts to the origin and/or selection of such mutants, due to prolonged infection and deficient immune response and T cell function. ${ }^{9}$ Generation of highly transmissible or pathogenic mutants in immunocompromised individuals could result from rapid viral evolution or saltational evolution, ${ }^{9}$ leading to new variants which can disseminate swiftly. Such patients need to be prioritised for immunisation and protected from SARS-CoV-2 infection. Treatment of such patients with monoclonal antibody or antiviral treatment may aid halting development of mutations and spread.

Treatment induced immunosuppression also leads to delayed viral clearance, ${ }^{10}$ potentially allowing continued viral replication and generation of mutants. Wang et al suggest caution, due to delayed viral clearance with no convincing improvement in patient survival after Corticosteroid use for treatment of COVID-19, in a meta-analysis of fifty-two trials involving 15710 patients.

Persistent SARS-CoV-2 infections could be the fuel for the ongoing epidemic and new variants, necessitating due attention to clinical conditions and treatments leading to delayed viral shedding.

\section{Ethics}

Samples were collected as part of routine testing for patients reporting to the Emergency Department at the All India Institute of Medical Sciences, New Delhi. The RNA extracted from the SARS-CoV-2 specimens were subjected to whole-genome sequencing following the IEC approvals (IEC-679/03.07.2020, RP-32/2020).

\section{Data Availability}

The sequence data is available in GISAID (https://www.gisaid.org) with IDs EPI_ISL_2426190EPI_ISL_2426253

\section{Conflict of Interest}

Authors declare no conflicts of interest.

\section{Acknowledgement}

Authors acknowledge funding from CSIR India through grant MLP005. Author MI acknowledge research fellowships from ICMR. Authors BJ, AVR, MKD, VG and AB acknowledge fellowship from CSIR India. The funders had no role in the preparation of the manuscript or decision to publish. COVID CBNAAT CORE GROUP includes contributions of P. V. Sowjanya, K V P Sai Kiran Kumar, Vishwanath Singh Yadav, Jawed Ahmed,Arkendu Basu, Aashirwad Panigrahy,Salmaan Khan, Amit Sehgal, Pragati Grover, V D Bamola, M Nizammuddin Ahmed, Sudesh Gaurav, 
Stephen Raj J, Raj Narayan, Sai Laxmi Mahapatra, Sruti Janani, Neha Nityadarshini, Jaya Biswas, Monika Sinha, Angitha K.P., Aswin Sasi Kumar, Bharat Chandra Das.

\section{References}

1. Tang X,Musa SS, Zhao S, et al. Reinfection or Reactivation of Severe Acute Respiratory Coronavirus 2: A systematic Review.Frnt. Public Health,2021;doi:0.3389/fpubh.2021. 663045

2. Bhoyar RC, Jain A, Sehgal P, et al. High throughput detection and genetic epidemiology of SARS-CoV-2 using COVIDSeq next-generation sequencing. PLoS One. 2021;16(2):e0247115.

3. Koboldt DC, Chen $\mathrm{K}$, Wylie T, et al. VarScan: variant detection in massively parallel sequencing of individual and pooled samples. Bioinformatics. 2009;25(17):2283-2285.

4. O'Toole Á, Scher E, Underwood A, et al. Assignment of Epidemiological Lineages in an Emerging Pandemic Using the Pangolin Tool. Virus Evolution. Published online 2021. doi:10.1093/ve/veab064

5. Choi B, Choudhary MC, Regan J, et al. Persistence and Evolution of SARS-CoV-2 in an Immunocompromised Host. N Engl J Med. 2020;383(23):2291-2293.

6. Rubingh J, van der Spek A, Fliers E, Boelen A. The Role of Thyroid Hormone in the Innate and Adaptive Immune Response during Infection. Compr Physiol. 2020;10(4):1277-1287.

7. Wang J, Kaperak C, Sato T et al.COVID-19 reinfection:a rapid systematic review of case reports and case series. J Investig Med 2021;69:1253-1255. Doi: 10.1136/jim-2021.001853.

8. Albillos A, Lario M, Álvarez-Mon M. Cirrhosis-associated immune dysfunction: distinctive features and clinical relevance. J Hepatol. 2014;61(6):1385-1396.

9. Corey L, Beyrer C, Cohen M S et al. SARS-CoV-2 Variants in Patients with Immunosuppression. NEJM 2021; 385(6):562-566.

10. Wang J, Yang W, Chen P, et al. The proportion and effect of corticosteroid therapy in patients with COVID-19 infection: a systematic review and meta-analysis. PLoS One 2021; doi.org/10.1371/journal.pone.0249481 